\title{
Alternative methods for solving nonlinear two-point boundary value problems
}

https://doi.org/10.1515/phys-2018-0050

Received December 2, 2017; accepted March 24, 2018

\begin{abstract}
In this sequel, the numerical solution of nonlinear two-point boundary value problems (NTBVPs) for ordinary differential equations (ODEs) is found by Bezier curve method (BCM) and orthonormal Bernstein polynomials (OBPs). OBPs will be constructed by Gram-Schmidt technique. Stated methods are more easier and applicable for linear and nonlinear problems. Some numerical examples are solved and they are stated the accurate findings.
\end{abstract}

Keywords: Nonlinear boundary value problems, orthonormal Bernstein.

PACS: 02.60.Cb; 02.60.Lj

\section{Introduction}

In this paper, the following NTBVPs are considered

$$
\begin{aligned}
& y^{\prime \prime}=f\left(x, y, y^{\prime}\right), \\
& l_{a} y(a)-m_{a} y^{\prime}(a)=k_{a}, x \in\left[x_{0}, x_{f}\right]=[a, b], a, b \in R, \\
& l_{b} y(b)+m_{b} y^{\prime}(b)=k_{b},
\end{aligned}
$$

where $f \in\left(C[a, b] \times R^{2}, R\right)$ is a nonlinear function, $l_{a}, m_{a}$, $l_{b}$, and $m_{b}$ are given positive constants and $k_{a}, k_{b}$ are given constants.

We consider the above NTBVPs which occur in applied mathematics, theoretical physics, engineering, control and optimization theory. By various researchers, different numerical methods have been proposed. Among these are the Adomian decomposition method (ADM) [1], He-Laplace method [2], Homotopy perturbation method (HPM) [3]. There are many methods for solving of twopoint boundary value problems ODEs.

Fateme Ghomanjani: Department of Mathematics, Kashmar Higher Education Institute Kashmar, Iran,

E-mail: fatemeghomanjani@gmail.com

*Corresponding Author: Stanford Shateyi: Department of Mathematics, University of Venda, P Bag X5050, Thohoyandou 0950,

South Africa, E-mail: stanford.shateyi@univen.ac.za
Additionally, many papers stated the BCM. In Ref. [5], authors utilized the BCM for solving delay differential equation (DDE) and optimal control of switched systems numerically. In Ref. [6], authors stated the utilization of BCM on some linear optimal control systems with pantograph delays. For solving the quadratic Riccati differential equation BCM is utilized (see Ref. [7]). In Ref. [8], authors presented BCM for solving singular nonlinear equations of Lane-Emden type. In this work, one may utilize BCM and orthonormal Bernstein polynomial (OBP) method for solving NTBVPs.

The Bernstein polynomials are not orthonormal therefore Gram-Schmidt technique could be utilized to construct OBP.

The organization of this paper is classified as follows: Problem statement is introduced in Section 2. Orthonormal Bernstein polynomials of arbitrary order are presented in Section 3. Numerical examples are solved in Section 4. Finally, Section 5 will give a conclusion briefly.

\section{Problem statement}

Our strategy is utilizing BCM to approximate the solutions $y(x)$ where $y(x)$ is given below. Define the Bezier polynomials of degree $n$ over the interval $\left[x_{0}, x_{f}\right]$ as follows:

$$
y(x)=\sum_{r=0}^{n} a_{r} B_{r, n}\left(\frac{x-x_{0}}{h}\right), x_{f}=1, x_{0}=0,
$$

where $h=x_{f}-x_{0}$, and

$$
B_{r, n}\left(\frac{x-x_{0}}{h}\right)=\left(\begin{array}{l}
n \\
r
\end{array}\right) \frac{1}{h^{n}}\left(x_{f}-x\right)^{n-r}\left(x-x_{0}\right)^{r},
$$

is the Bernstein polynomial of degree $n$ over the interval $\left[x_{0}, x_{f}\right], a_{r}$ represents the control points. Suppose that $M_{B}$ is the coefficient matrix of $B_{r, n}(x), r=0,1, \ldots, n$, where $M_{B}(i, j)$ is the coefficient of the $B_{i, n}(x)$ with respect to the monomial $x^{j-1}$, then by Eq. (3) one may have

$$
\begin{gathered}
M_{B}(i, j)=(-1)^{i+j}\left(\begin{array}{c}
n \\
i
\end{array}\right)\left(\begin{array}{c}
n-i \\
j-i
\end{array}\right), \\
i=0,1, \ldots, n, j=i, \ldots, n,
\end{gathered}
$$


By substituting $y(x)$ in Eq. (1), one may define $R_{1}\left(x, a_{0}, a_{1}, \ldots, a_{n}\right)$ for $x \in\left[x_{0}, x_{f}\right]$ as follows:

$$
R_{1}\left(x, a_{0}, a_{1}, \ldots, a_{n}\right)=y^{\prime \prime}-f\left(x, y, y^{\prime}\right) \text {. }
$$

The convergence of this method is proven where $n \rightarrow \infty$ (see [9]).

Now, we define the residual function over the interval $\left[x_{0}, x_{f}\right]$ as follows

$$
R=\int_{x_{0}}^{x_{f}}\left(R_{1}\left(x, a_{0}, a_{1}, \ldots, a_{n}\right)\right)^{2} d x
$$

\section{Orthonormal Bernstein}

\section{polynomials of arbitrary order}

In this section, OBP is constructed. For arbitrary $n$, Eq. (6) can be obtained by induction. Now, let $n=3$, for simplicity $B_{i}=B_{i}(x)(i=0,1,2,3)$ and $O B_{i}=O B_{i}(x)$, hence

$$
\begin{aligned}
\psi_{0} & =B_{0}, O B_{0}=\frac{\psi_{0}}{\left\|\psi_{0}\right\|}, \\
\psi_{1} & =B_{1}-<B_{1}, O B_{0}>O B_{0}, O B_{1}=\frac{\psi_{1}}{\left\|\psi_{1}\right\|}, \\
\psi_{2} & =B_{2}-<B_{2}, O B_{0}>O B_{0}-<B_{2}, O B_{1}>O B_{1}, \\
O B_{2} & =\frac{\psi_{2}}{\left\|\psi_{2}\right\|}, \\
\psi_{3} & =B_{3}-<B_{3}, O B_{0}>O B_{0}-<B_{3}, O B_{1}>O B_{1} \\
& -<B_{3}, O B_{2}>O B_{2}, \\
O B_{3} & =\frac{\psi_{3}}{\left\|\psi_{3}\right\|},
\end{aligned}
$$

where $O B_{i}(i \geq 0)$ can be obtained from Gram-Schmidt orthonormalization technique

$$
\begin{aligned}
\psi_{m} & =B_{m}-<B_{m}, O B_{0}>O B_{0} \\
& -<B_{m}, O B_{1}>O B_{1}-<B_{m}, O B_{2}>O B_{2} \\
& -\ldots-<B_{m}, O B_{m-1}>O B_{m-1}, \\
O B_{m} & =\frac{\psi_{m}}{\left\|\psi_{m}\right\|}, \text { (for arbitrary m), }
\end{aligned}
$$

and

$$
\left\|\psi_{0}\right\|^{2}=<\psi_{0}, \psi_{0}>=\int_{0}^{1} \psi_{0} \psi_{0} d x,
$$

hence

$$
\begin{aligned}
& O B_{0}=8\left(\frac{1}{2}-t\right)^{3} \sqrt{7} \\
& O B_{1}=\left(\frac{1}{3}\left(24 t\left(\frac{1}{2}-t\right)^{2}+24\left(\frac{1}{2}-t\right)^{3}\right)\right) \sqrt{5}
\end{aligned}
$$

$$
\begin{aligned}
O B_{2} & =\left(\frac { 5 } { 6 } \left(24 t^{2}\left(\frac{1}{2}-t\right)+48 t\left(\frac{1}{2}-t\right)^{2}\right.\right. \\
& \left.\left.-\left(\frac{48}{5}\right)\left(\frac{1}{2}-t\right)^{3}\right)\right) \sqrt{3}, \\
O B_{3} & =12 t^{3}+36 t^{2}\left(\frac{1}{2}-t\right)+16 t\left(\frac{1}{2}-t\right)^{2}-8\left(\frac{1}{2}-t\right)^{3},
\end{aligned}
$$

then the following OBP is considered as

$$
y(x)=\sum_{i=0}^{n} a_{r} O B_{i}(x), x \in[0,1],
$$

by substituting Eq. (7) in Eq. (4), we can continue the stated algorithm for solving NTBVP.

\section{Numerical examples}

Now, some numerical examples of NTBVPs are stated to illustrate BCM. All results are obtained by utilizing Maple 14.

Example 1. First, the following NTBVP is considered (see [4])

$$
\begin{aligned}
y^{\prime \prime}= & -\cos y \sin y^{\prime}+2 y+\cos \left(1-x^{2}\right) \sin (2 x) \\
- & 2\left(x^{2}-1\right)+2, \quad x \in[-1,1], \\
& y(-1)=0, y(1)=0, \\
& y_{\text {exact }}=x^{2}-1,
\end{aligned}
$$

Applying the proposed technique with $n=4$, one may have

$$
\begin{aligned}
y_{\text {approx }} & =8.807584016 \times 10^{-309}(1-x)^{4} \\
& -(0.250000000000226(x+1))(1-x)^{3} \\
& -0.500000000000764(x+1)^{2}(1-x)^{2} \\
& -0.249999999994255(x+1)^{3}(1-x),
\end{aligned}
$$

where the obtained error is zero. The graphs of approximated and exact solution $y$ are plotted in Figure 1.

Example 2. Second, one may solve the Example 1 by $O B P$. Let $n=3$, we can obtain easily the following results (see Table 2). these results can be obtained by OBP method.

$$
y(x)=-1+4 \times 10^{-9} x+x^{2}+2 \times 10^{-9} x^{3}, x \in[0,1] .
$$

Example 3. Third, the following NTBVP is considered (see [4])

$$
\begin{aligned}
y^{\prime \prime} & =-\sin y^{\prime}-\cos y+\cos x\left(4 x^{2}-1\right)-\sin \left(1-12 x^{2}\right) \\
& +24 x, \quad x \in\left[-\frac{1}{2}, \frac{1}{2}\right]
\end{aligned}
$$


Table 1: The error of proposed method for Example 1

\begin{tabular}{cc}
\hline$x$ & error of proposed method \\
\hline 0.0 & 0.0 \\
0.1 & $2.795541576 \times 10^{-13}$ \\
0.2 & $9.436895709 \times 10^{-14}$ \\
0.3 & $8.264500195 \times 10^{-13}$ \\
0.4 & $2.513322883 \times 10^{-12}$ \\
0.5 & $4.755085214 \times 10^{-12}$ \\
0.6 & $7.098766019 \times 10^{-12}$ \\
0.7 & $8.850919997 \times 10^{-12}$ \\
0.8 & $9.076406293 \times 10^{-12}$ \\
0.9 & $6.598610547 \times 10^{-12}$ \\
1.0 & 0.0 \\
\hline
\end{tabular}

$$
\begin{aligned}
& y\left(-\frac{1}{2}\right)-y^{\prime}\left(-\frac{1}{2}\right)=-2, \\
& y\left(\frac{1}{2}\right)+y^{\prime}\left(\frac{1}{2}\right)=2, \\
& y_{\text {exact }}=x\left(4 x^{2}-1\right),
\end{aligned}
$$

Applying the proposed technique with $n=4$, one may have

$$
\begin{aligned}
y_{\text {approx }} & =-2.22044604925031 \times 10^{-16}\left(\frac{1}{2}-x\right)^{4} \\
& +\left(2\left(x+\frac{1}{2}\right)\right)\left(\frac{1}{2}-x\right)^{3} \\
& -1.33226762955019 \times 10^{-15}\left(x+\frac{1}{2}\right)^{2}\left(\frac{1}{2}-x\right)^{2} \\
& -2\left(x+\frac{1}{2}\right)^{3}\left(\frac{1}{2}-x\right) .
\end{aligned}
$$

where the obtained error is stated in Table. 3 for $n=3$ and $n=4$. This shows that the error should decay as $n$ increases. The graphs of approximated and exact solution $y$ are plotted in Figure 2 .

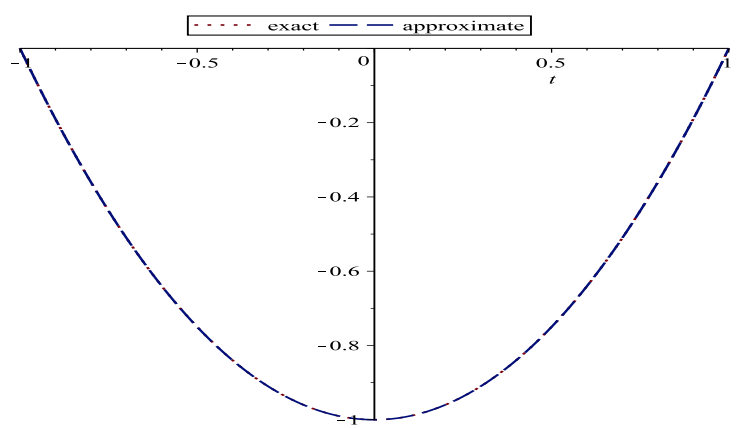

Figure 1: The graphs of approximated and exact solution $y$ for Example 1
Table 2: The error of OBP method for Example 2

\begin{tabular}{cc}
\hline$x$ & error of OBP method \\
\hline 0.0 & 0.0 \\
0.1 & 0.0 \\
0.2 & 0.0 \\
0.3 & 0.0 \\
0.4 & 0.0 \\
0.5 & 0.0 \\
0.6 & $1 \times 10^{-10}$ \\
0.7 & $1 \times 10^{-10}$ \\
0.8 & 0.0 \\
0.9 & 0.0 \\
1.0 & 0.0 \\
\hline
\end{tabular}

Table 3: The error of proposed method with $n=3,4$ for Example 3

\begin{tabular}{ccc}
\hline$x$ & error with $n=4$ & error with $n=3$ \\
\hline 0.0 & $2.220446049 \times 10^{-16}$ & $2.220446049 \times 10^{-16}$ \\
0.1 & $1.942890293 \times 10^{-16}$ & $1.665334537 \times 10^{-16}$ \\
0.2 & $2.498001805 \times 10^{-16}$ & $1.110223025 \times 10^{-16}$ \\
0.3 & $2.498001805 \times 10^{-16}$ & $2.775557562 \times 10^{-17}$ \\
0.4 & $2.081668171 \times 10^{-16}$ & $1.387778781 \times 10^{-17}$ \\
0.5 & $1.804112415 \times 10^{-16}$ & $1.110223025 \times 10^{-16}$ \\
0.6 & $1.526556659 \times 10^{-16}$ & $1.804112415 \times 10^{-16}$ \\
0.7 & $8.326672685 \times 10^{-17}$ & $1.942890293 \times 10^{-16}$ \\
0.8 & $5.551115123 \times 10^{-17}$ & $1.110223025 \times 10^{-16}$ \\
0.9 & $2.775557562 \times 10^{-17}$ & $5.551115123 \times 10^{-17}$ \\
1.0 & 0.0 & $2.220446049 \times 10^{-16}$ \\
\hline
\end{tabular}

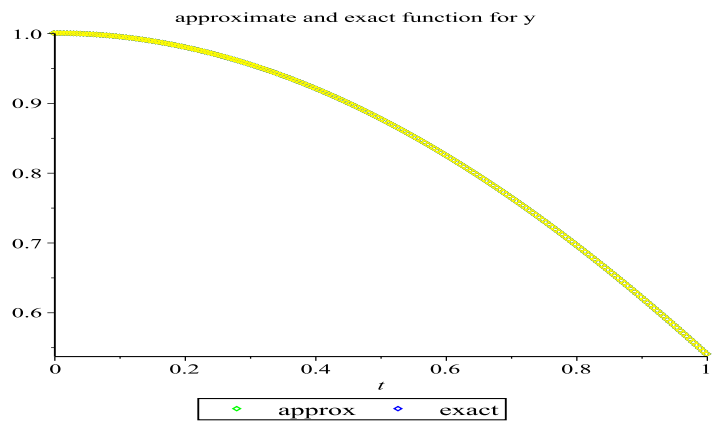

Figure 2: The graphs of approximated and exact solution $y$ for Example 3

\section{Conclusions}

This paper deals with the approximate solution of NTBVPs via BCM and OBP. The unity of the function of orthogonality for Bernstein polynomials (BPs) and the simplicity of applying BPs are great merits that make the technique very attractive and easy to use. Illustrative examples are solved 
to express the validity and applicability of the proposed methods. The approximate solution is coming very close to the exact solution.

Conflict of Interests: The author declares that there is no conflict of interest in the use of the above mentioned software.

Acknowledgement: The authors would like to thank the anonymous reviewers of this paper for their careful reading, constructive comments and nice suggestions, which have improved the paper very much. The Second author wishes to acknowledge financial support from the University of Venda.

\section{References}

[1] Wazwaz A.M., Adomian decomposition method for a reliable 5 treatment of the Bratu type equations, Appl. Math. Comp., 2005, $166,652-663$.
[2] Mishra H.K., He-Laplace method for the solution of two-point boundary value problems, Amer. J. Math. Anal., 2014, 2(3), 45-49.

[3] Xu L., He's homotopy perturbation method for a boundary layer equation in unbounded domain, Comp. Math. Appl., 2007, 54, 1067-1070.

[4] Cuomo S., Marasco A., A numerical approach to nonlinear twopoint boundary value problems for ODEs, Comp.Math. Appl., 2008, 55, 2476-2489.

[5] Ghomanjani F., Farahi M.H., Optimal control of switched systems based on bezier control points, Int. J. Intell. Syst. Appl., 2012, 4(7), 16-22.

[6] Ghomanjani F., Farahi M.H., Kamyad A.V., Numerical solution of some linear optimal control systems with pantograph delays, IMA J. Math. Control Inf., 2015, 32(1), 225-243.

[7] Ghomanjani F., Khorram E., Approximate solution for quadratic Riccati differential equation, J. Taibah Univ. Sci., 2017, 11 246250.

[8] Adiyaman M., Oger V., A residual method using Bezier curves for singular nonlinear equations of Lane-Emden type, Kuwait J. Sci., 2017, 44(4), 9-18.

[9] Ghomanjani F., Farahi M.H., Gachpazan M., Bezier control points method to solve constrained quadratic optimal control of time varying linear systems, Comp. Appl. Math., 2012, 31(3), 1-24. 